\title{
Un fonds patrimonial dans une bibliothèque d'ingénieurs
}

Entretien avec Catherine Kounelis, directrice de la bibliothèque de l'ESPCI

\section{Christian Jacob}

\section{CpenEdition}

\section{Journals}

Édition électronique

URL : https://journals.openedition.org/rbnu/1637

DOI : $10.4000 /$ rbnu. 1637

ISSN : 2679-6104

Éditeur

Bibliothèque nationale et universitaire de Strasbourg

\section{Édition imprimée}

Date de publication : 1 novembre 2014

Pagination : 56-67

ISBN : 9782859230548

ISSN : 2109-2761

\section{Référence électronique}

Christian Jacob, « Un fonds patrimonial dans une bibliothèque d'ingénieurs », La Revue de la BNU [En ligne], 10 | 2014, mis en ligne le 01 novembre 2014, consulté le 18 mai 2021. URL : http:// journals.openedition.org/rbnu/1637 ; DOI : https://doi.org/10.4000/rbnu.1637

\section{(c) (i) (2)(2)}

La Revue de la BNU est mise à disposition selon les termes de la Licence Creative Commons Attribution - Pas d'Utilisation Commerciale - Partage dans les Mêmes Conditions 4.0 International. 


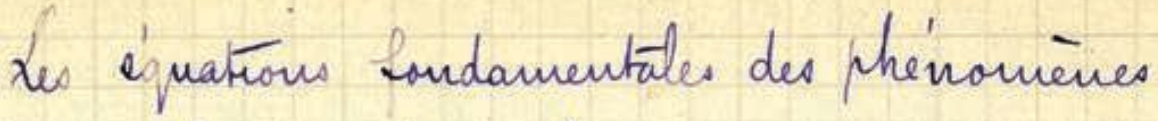

\section{Selectromagnetiques daus les cosps en mourvenent.}

par Hermann Minkowski

\section{1 introduction}

L'aund n'ent has creore complet actuellement tur les



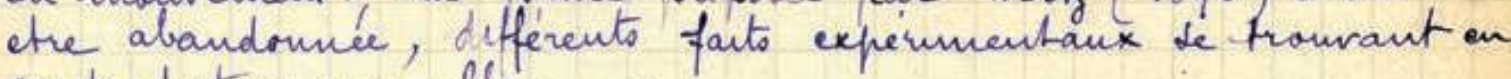
contradiction aver elle.

Bascie cur une représentation atomidiane de

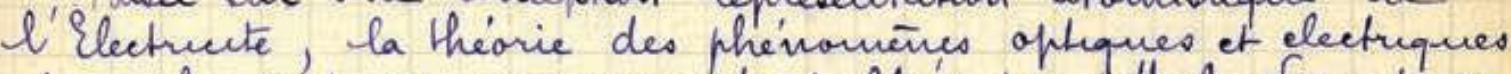

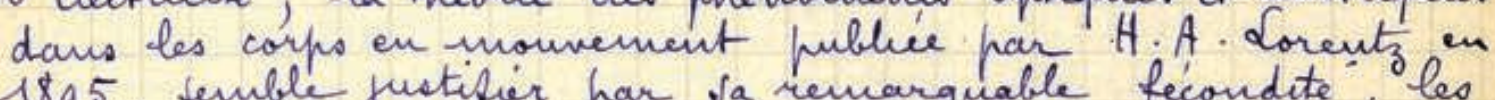

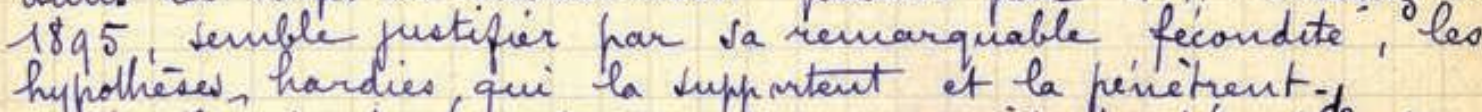
da. Theorie de dorent

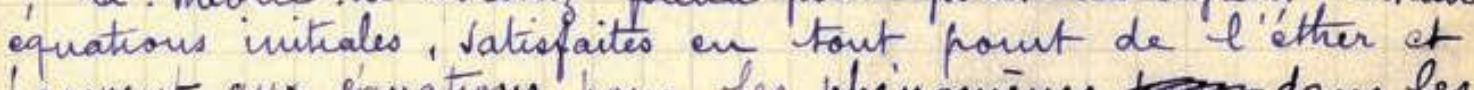
hannent ane escrations hour les thenomines dans bes



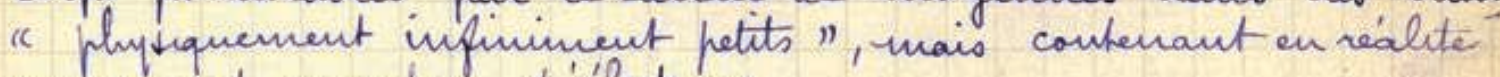
im reand, nowive d'électrons

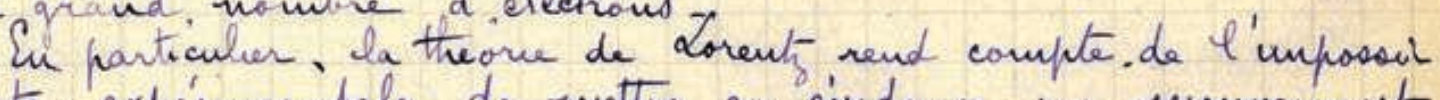

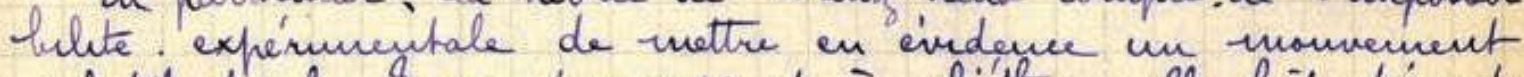
relatif de la Geve par raptrot a l'ather, elle fait dependre ee resultat d'une covarance des equationis minteales four

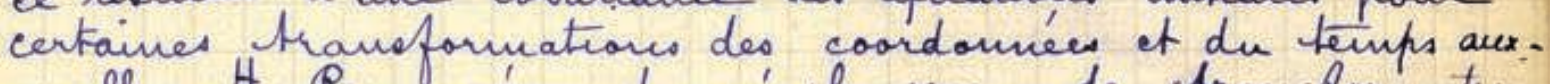

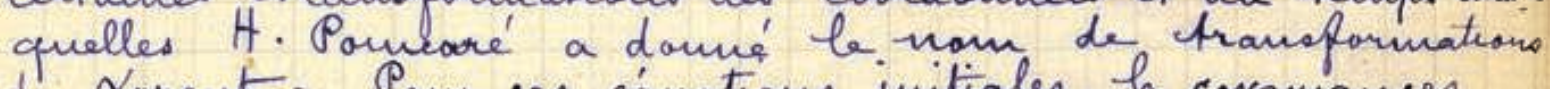
de corento. Paur ces equations initiales, a covarames

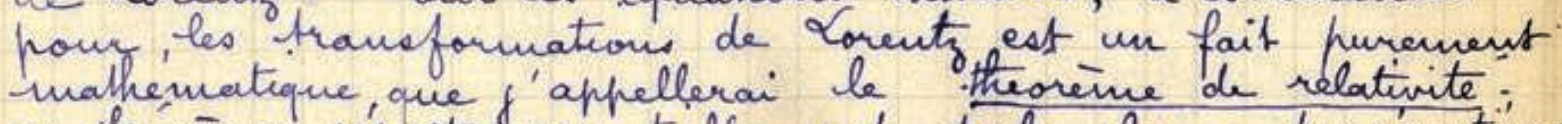

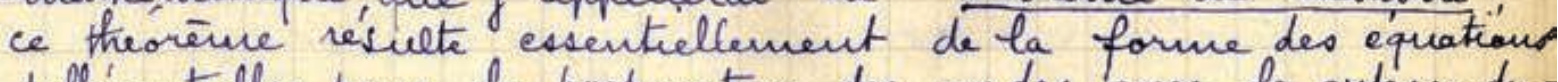


la himiere.

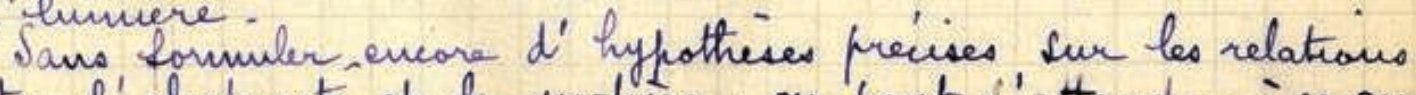

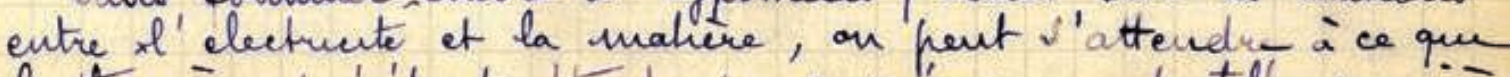

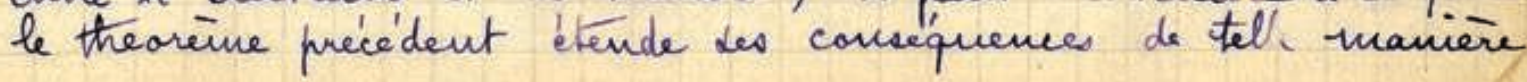




\title{
Un fonds patrimonial
}

\section{dans une bibliothèque d'ingénieurs}

\author{
Entretien avec Catherine Kounelis, \\ directrice de la bibliothèque de l'ESPGI
}

Une bibliothèque est à l'image de son public. Dans une école d'ingénieurs, elle est très différente d'une bibliothèque de lecture publique. Mais elle est aussi différente d'une bibliothèque universitaire. A l'École supérieure de physique et de chimie industrielles de la Ville de Paris (ESPCI ParisTech), il y a une forte interaction entre enseignement et recherche, et cette spécificité se reflète dans sa bibliothèque. Ajoutez maintenant une composante patrimoniale, et vous aurez les ingrédients qui composent le profil de la bibliothèque de l'ESPCI.

\section{Catherine Kounelis, pouvez-vous nous présenter votre propre parcours : comment êtes-vous arrivée à la bibliothèque de l'ESPCI ?}

J'ai une formation initiale en chimie et en histoire des sciences ; je me suis rapprochée de l'ESPCI car j'ai été attirée par son histoire. Ses fondateurs et ses trois premiers directeurs étaient des chimistes alsaciens. Aujourd'hui, il y a de nombreux travaux d'historiens sur les liens de la chimie ${ }^{1}$ avec l'Alsace et il n'y a plus d'originalité à en parler. Il y a vingt ans cependant, il s'agissait d'un tout nouveau champ de recherche; je l'ai vu émerger et évoluer, j'ai assisté aux premières rencontres scientifiques autour de Bernadette BensaudeVincent, de Georges Bram, de Danielle Fauque et de Jean Jacques au Club d'histoire de la chimie. Ensuite, après mon arrivée à l'ESPCI en 1998, j'ai découvert l'œuvre extraordinaire et la personnalité de Paul Langevin, ce qui m’a rapprochée de l'histoire de la physique.

\section{L'ESPCI est à la fois une grande école scientifique, très intégrée dans le paysage de l'enseignement français, et un lieu d'histoire, un lieu de mémoire...}

Il faut rappeler le contexte dans lequel l'ESPCI a été créée : c'est à la suite de la guerre de 1870, quand la France veut investir dans l'éducation, considérée comme l'un des piliers de la reconstruction du pays. La Ville de Paris participe à cet effort national par la création d'une dizaine d'écoles professionnelles, parmi lesquelles l'École Diderot, l'École Boulle, l'École Estienne. Elles devaient donner une perspective d'études avancées à des élèves sortis des écoles primaires supérieures, et qui n'avaient pas les moyens financiers de continuer dans un lycée ou dans une grande école. L'École municipale de physique et de chimie industrielles (EMPCI) - elle devint " supérieure " en 1948 - avait à l'origine un statut d'école professionnelle, mais elle s'en est détachée par son haut niveau d'enseignement scientifique et l'âge d'entrée, de 16 à 19 ans, contre 13 à 16 ans pour les autres écoles professionnelles. La volonté de la Ville de Paris de participer à l'effort national pour l'éducation des jeunes rejoignait le projet de chimistes industriels alsaciens (dont Paul Schützenberger et Charles Lauth, nés à Strasbourg) de créer un établissement pour préparer les jeunes gens aux carrières industrielles. D'où l'une des spécificités de l'École qui est l'alliance forte entre science et industrie. Il faut rappeler qu'après la guerre de 1870 , quand Mulhouse, où il y avait une école de chimie, fut annexée par l'Allemagne, il n'y avait plus en France d'enseignement de chimie orienté vers l'industrie et ses applications. C'est ce qu'a fait valoir Charles Lauth, par ailleurs ancien conseiller de Paris, quand il a soumis au Conseil de Paris sa proposition de création de l'EMPCI. 


\section{Comment définiriez-vous la bibliothèque de l'École aujourd'hui ? Une bibliothèque de recherche ou d'enseignement?}

Avec ses 400 mètres carrés et ses 75 places, la salle de lecture peut accueillir les 400 élèves ingénieurs, sauf pendant les examens où les places deviennent plus recherchées. Nous manquons cependant beaucoup d'espaces de conservation des collections anciennes. Les horaires d'ouverture ont été prolongés récemment jusqu'à $22 \mathrm{~h}$ le soir, ce qui est plutôt rare pour ce type de bibliothèque. Les habitudes de consultation ont aussi évolué. Il y a quinze ans, la direction des études nous demandait de distribuer tous les livres d'enseignement en début d'année scolaire. Les élèves étant répartis en 18 groupes de travaux pratiques, on achetait les livres par séries de 9 ou de 18 pour en prêter un par groupe ou à un groupe sur deux. Aujourd'hui, les élèves passent plus de temps en salle de lecture. L'équipement informatique de la salle (postes en libre accès) a aussi modifié un peu le paysage. Mais cela n'est rien comparé à l'évolution de la documentation destinée aux chercheurs.

Financièrement parlant, aujourd'hui plus de $80 \%$ du budget de la documentation est consacré à la recherche, en raison des coûts très élevés des abonnements aux revues spécialisées. Lors de mon entrée en fonction, il y a quinze ans, c'était déjà le cas. C'est la raison pour laquelle la bibliothèque a organisé ces dernières années une journée et des ateliers pour promouvoir l'open access " équitable ", c'est-à-dire le dépôt des articles dans une archive ouverte institutionnelle où ils peuvent être consultés en libre accès (voie verte), la publication selon le modèle auteur-payeur (voie dorée), mais à des frais de publication raisonnables, à moins de 1000 \$ par article, ou encore le développement d'autres initiatives, comme les épi-revues ${ }^{2}$.

Aujourd'hui, ce sont majoritairement les élèves qui sont dans la salle de lecture, car les chercheurs ont désormais accès aux ressources numériques depuis leurs postes de travail. Les étudiants trouvent les livres en libre accès et ils peuvent bien sûr les emprunter. Un atelier de reprographie fonctionne même à l'intérieur des murs de la bibliothèque, permettant aux élèves de photocopier et de relier leurs notes de cours et les rapports de stage. Nous faisons très attention à mettre à leur disposition tous les livres dont ils ont besoin pour l'enseignement. Nous sommes donc une bibliothèque d'enseignement et de recherche, sans qu'on puisse dire que l'une de ces deux composantes l'emporte sur l'autre.

\section{Est-ce que la nature des documents et les comportements des usagers ont beaucoup évolué ces dernières années?}

J'ai pris mes fonctions ici en 1998 lors de l'apparition dans le paysage français de l'IST ${ }^{3}$ des premiers périodiques électroniques. Ce fut une révolution. On ne savait pas que d'autres révolutions nous attendaient, comme celle du " big deal " (la commercialisation des revues par bouquets) quelques années plus tard et celle de l'open access plus récemment. On a vu les premiers modèles tarifaires émerger et se consolider au fil du temps. On a surtout vu les chercheurs déserter notre salle de lecture. Fini le présentoir à l'entrée, sur lequel on étalait les derniers fascicules des périodiques reçus. Personne ne venait plus les consulter. On nous disait que la documentation électronique allait générer des économies, puisqu'on supprimait le papier - ce qu'aucun bibliothécaire n'a jamais cru un seul instant. En réalité, le « big deal » a fait exploser les coûts. Tous les collègues dans les bibliothèques de recherche vous diront la même chose. En quinze ans, le métier de bibliothécaire s'est complètement transformé. L'activité de la bibliothèque est devenue beaucoup moins visible, puisque la consultation passe par les réseaux informatiques - le nombre des téléchargements en constante augmentation en est la preuve. Le chercheur a aujourd'hui accès à une énorme quantité d'informations, à une vitesse qu'il n'imaginait pas il y a vingt ans. L'accélération du rythme des progrès scientifiques est sans doute liée aux chercheurs aujourd'hui plus nombreux sur la planète, mais aussi au fait qu'un chercheur qui débute une nouvelle recherche peut constituer un corpus 
sil'on refu'teute far $r$ le vedeen d'univero que a four couporants $x_{1} x_{2} x_{3} x_{4}$ on a

$$
m_{0} \frac{d^{2} r}{d \tau^{2}}=K
$$

$K$ ent herfars a' $\frac{d r}{d t}$ el on feut former le fro suit sealane geviralive a'4faram.

$$
K \frac{d r}{d h}=0
$$

On a $\partial^{\prime}$ cilleurs $m_{0}=\frac{E_{0}}{c^{2}}$

udentifuls Inotion, celles o'energi et \& mane.

Affication. Nians calaule' inestie d'une'action

$$
m_{0}=\frac{2 \mu_{0} e^{2}}{3 a}
$$

l'energie elecho stat eit

$$
\frac{1}{2} \frac{Q^{2}}{K C}=\frac{e^{2}}{2 K_{0} a}
$$

Xnovs a falle aimetre une fuenion daus b hilien eunromant (Ponicare'). L'enery. dee far mute' devolume est

$$
\frac{e^{2}}{8 \pi k_{0} a^{4}}
$$

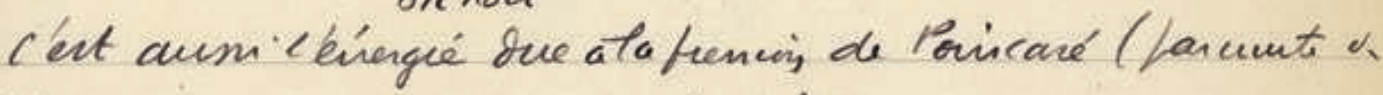
volumal on mulefer tar $\frac{4}{3} \pi a^{3}$

l'mergè to tale

$$
\frac{e^{2}}{2 K_{0} a}+\frac{e^{2}}{6 K_{0} a}
$$

Joù $\quad E_{0}=\frac{2 e^{2}}{3 K_{0} a}$

etfon a $m_{0}=\frac{E_{0}}{c^{2}}=\frac{2 \mu_{0} e^{e}}{3 a}$

Cours de Paul Langevin au Collège de France sur la relativité, 1910-1911.

Notes prises par Jean Saphores (coll. ESPCI).

59 


\section{Letinomètre de 16 . Téry \\ Constante solavie, bemperative du soleit.}

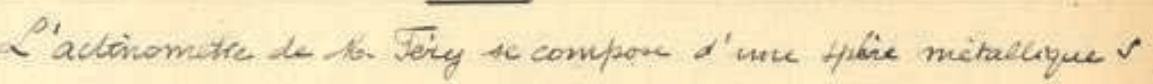
peisutant unce ouverture 0 . destincie a permette


forme prar un verre dipoli. An centre de la pplière ts

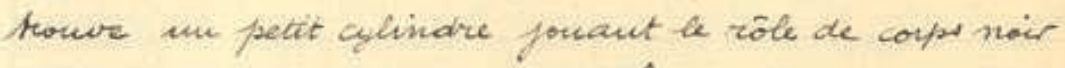
wie' d'une part a' un fit de fer evcommunicaheri avec la sphire etáautrepart à in fib a constantan esole' Clectriquement de la tphere. Les deux bornes set $B^{\prime}$ Sont relici a un millivotmitre of qui lowaue le copps $e$ est froit se troute au ziro. Li l'on vaciet a'placer deount l'owerture o une sowra chande

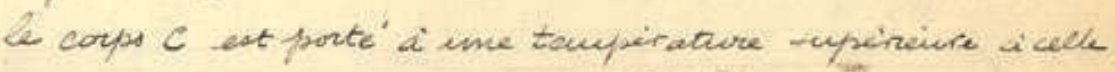
dela mars mitullique et une force élechomohice thermo électrique frend naviance daus le curicut du mullivotmelre lu' donnaut une certurie dévianon'. Cete divianon commene par croitre eutoment pui deveint constanto lowgre la chaleur gugnie parte corne estegah à la chalur qu'il jerd par rayomenneut, convecioni et conductiblité. De cette dióiahoin ou put apnis unetulormage préalabe deduire la quautet de chaleur rayomne par da source chaude vers le coups e par inite' detcups. Etalonnage de l'actinomètre. A $L^{\prime}$ initirieur du corps $C$ se trouve un curcuit relié à deux bornes b At b' sovis de laparar de la spheire lorsque ce fir est parcouru par in couraut

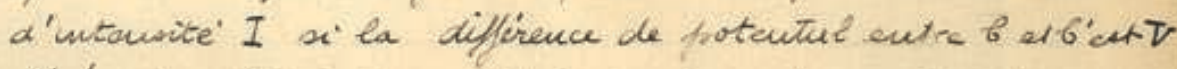
l'évergie disipie par effot foule dow hercuit est prar reconde VI parcossiquent facile adéterminer an moyund im galianomete et d'un voetmitre. Cetce inergie culorifigure tert a'changls le corps $C$

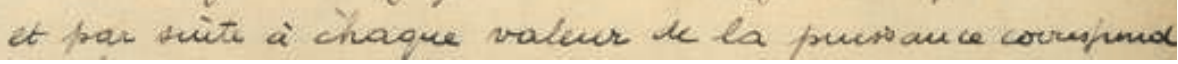




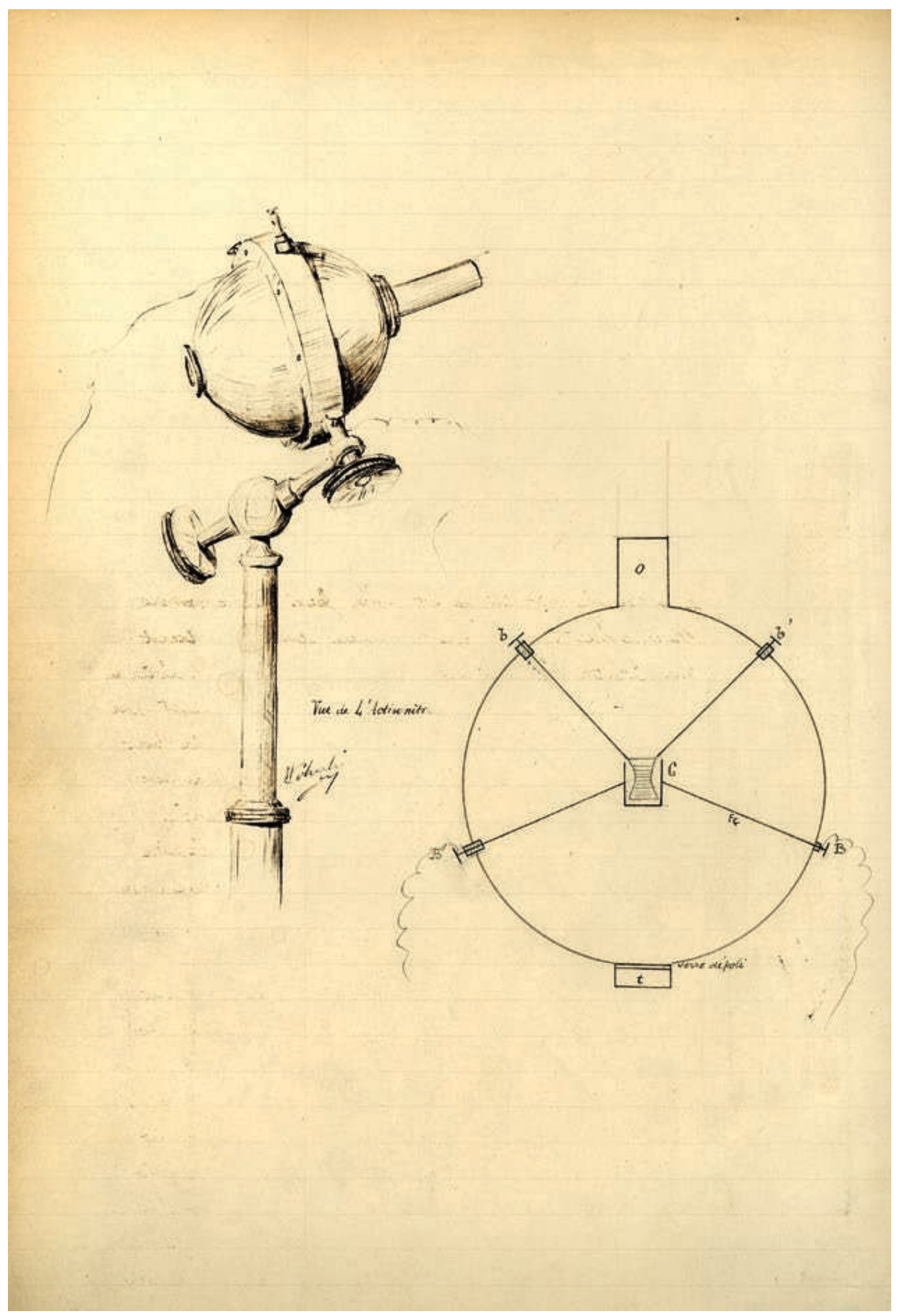

Cahier d'expériences. Manipulations de physique par les élèves de Charles Féry (1865-1935), professeur d'optique à l'EMPCI (coll. ESPCI). 


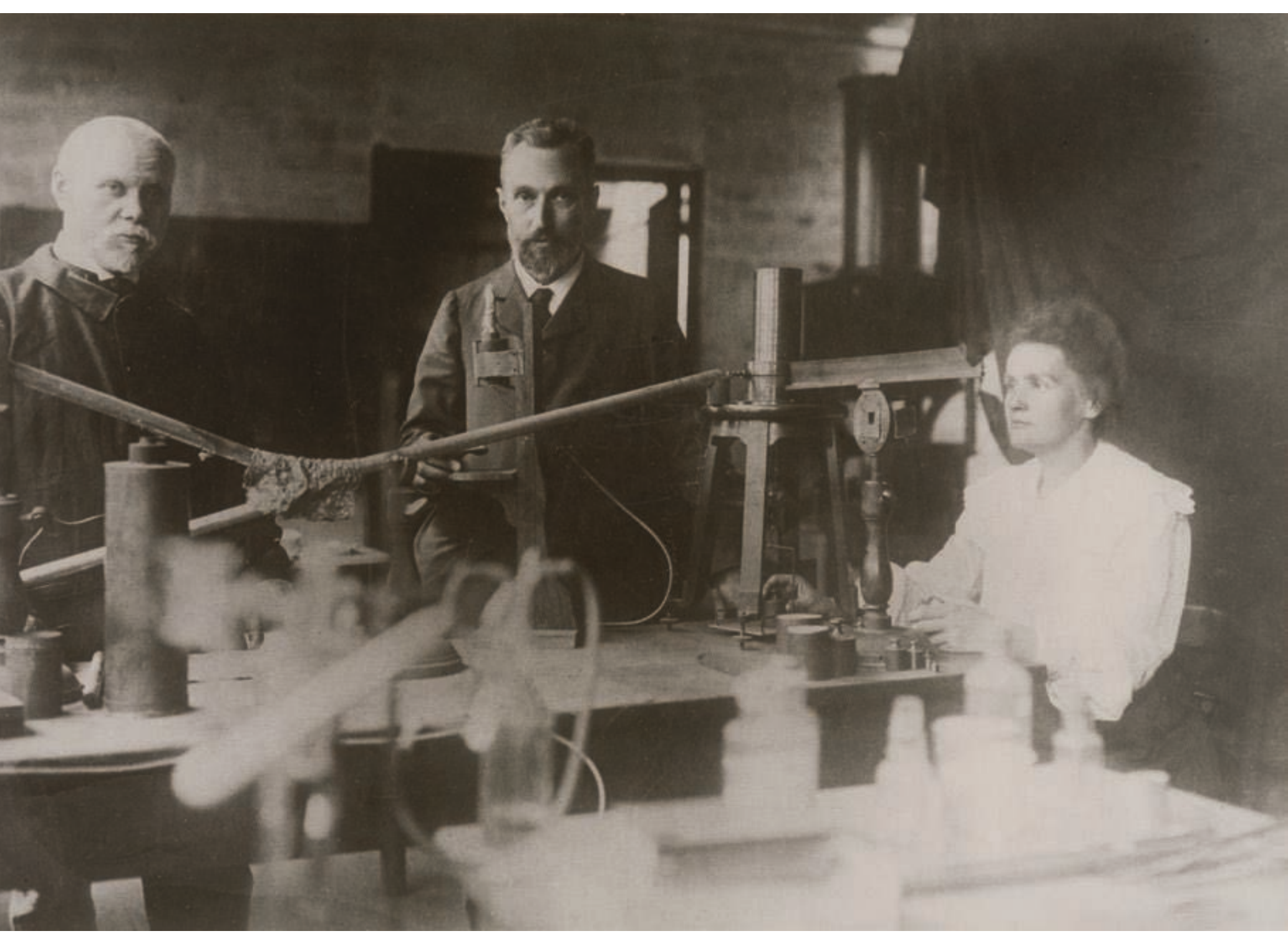

Pierre et Marie Curie dans le laboratoire de l'École de physique et de chimie industrielles, où ils ont découvert le radium et le polonium en 1898 (coll. ESPCI). 
bibliographique en quelques clics, là où il devait consacrer des semaines, parfois des mois, à se déplacer dans différentes bibliothèques pour trouver un article et faire des photocopies.

Un trait particulier de ces mutations réside dans l'éclipse des encyclopédies, des grandes collections sur la chimie organique, sur les matériaux, sur la chimie minérale. Ces ouvrages de référence étaient les précurseurs des bases de données ou des encyclopédies en ligne d'aujourd'hui. Cela fait longtemps que nous n'achetons plus ce genre de collections. Et celles que nous avons conservées ne sont plus consultées. Les chercheurs recherchent d'abord les informations sur Internet, mais s'ils ne les y trouvent pas - car contrairement à ce qui se dit parfois, tout n'est pas encore en format électronique -, ils pensent rarement à se rendre dans la bibliothèque pour les consulter en format papier. C'est pourquoi il est essentiel que les catalogues des bibliothèques aient une bonne visibilité auprès des chercheurs, et qu'ils intègrent sur le même outil informatique, si possible, et les ressources électroniques et les ressources disponibles en format papier.

\section{Pourrait-on écrire une histoire de la physique et de la chimie en France ou en Europe à partir des collections de la bibliothèque ? Est-ce que la bibliothèque a suivi l'évolution générale et avec quel degré de latence, ou est-ce qu'elle a privilégié des aspects particuliers du développement scientifique ?}

Je ne peux pas répondre de manière générale. Dans le cas de l'ESPCI, les archives nous révèlent qu'en 1888, en plus de son abonnement en cours, la bibliothèque avait fait l'acquisition rétrospective de 212 volumes reliés des Annales de Physique et de Chimie de la période 1789-1862. Cela apporte une indication sur la typologie des ressources que les chercheurs ont besoin de consulter à un moment donné de l'Histoire. Pour la période récente que j'ai connue, je peux affirmer que la bibliothèque a suivi les besoins des utilisateurs, les exigences de la science et de son enseignement. On le voit dans les évolutions du catalogue des périodiques : il y a des abonnements que l'on arrête, d'autres que l'on commence pour des raisons d'évolution des disciplines, même si nous nous efforçons de préserver une certaine continuité, car il est important de constituer des collections sans lacunes. Dans les dernières années, les politiques commerciales de quelques grands éditeurs scientifiques tendent cependant à imposer un socle de ressources documentaires commun à tous les établissements et à diminuer ce faisant les parts de budget que les bibliothèques peuvent consacrer aux ressources fournies par les plus petits éditeurs. Cela conduit malheureusement à réduire la diversité des ressources bibliographiques dans l'écosystème de l'IST.

Pour la politique d'acquisition des ouvrages, c'est différent : il est fréquent d'assister à l'accueil dans l'établissement de nouvelles équipes de recherche, et même à l'émergence d'une nouvelle branche scientifique. C'est le cas récemment de la microfluidique, nouvelle discipline prometteuse pour laquelle l'ESPCI vient d'inaugurer les travaux d'aménagement d'un bâtiment destiné à accueillir un centre de recherche de réputation mondiale. Nous adaptons alors notre offre documentaire à ces besoins. De même, on tient compte des matières et des programmes enseignés, et les achats suivent cette évolution. Par exemple, lorsque Pierre-Gilles de Gennes a introduit la biologie à l'ESPCI en 1994, nous avons dès lors consolidé notre collection en biologie, le fonds ancien de l'École étant surtout axé sur la physique et la chimie. On a peu de mathématiques, presque rien en médecine. La vocation pédagogique et scientifique première de l'École a certainement modelé les collections. Les enseignants-chercheurs nous font d'ailleurs souvent des suggestions d'achats pour les matières dont ils ont la charge d'enseignement, ou pour des livres de recherche.

\section{Est-ce que vous conservez la totalité de votre fonds, même les livres les plus anciens, qui peuvent être aujourd'hui dépassés sur le plan scientifique? Est-ce qu'il y a une réactualisation permanente de la collection?}

Nous pratiquons, comme nombre de bibliothèques, le désherbage, et chaque bibliothèque a sa propre politique selon sa mission et selon les besoins de ses usagers. Des centaines de livres dépassés sur le plan scientifique ont été retirés des étagères au fil du temps. Cependant, nous faisons attention à garder certains livres anciens, notamment les livres en langue anglaise (les livres français sont de toute façon conservés par le biais du dépôt légal) et à conserver, voire acquérir dans certains cas, des ouvrages anciens écrits par des professeurs et des ingénieurs de l'ESPCI. Cette partie du fonds ancien participe de l'histoire de l'École. 

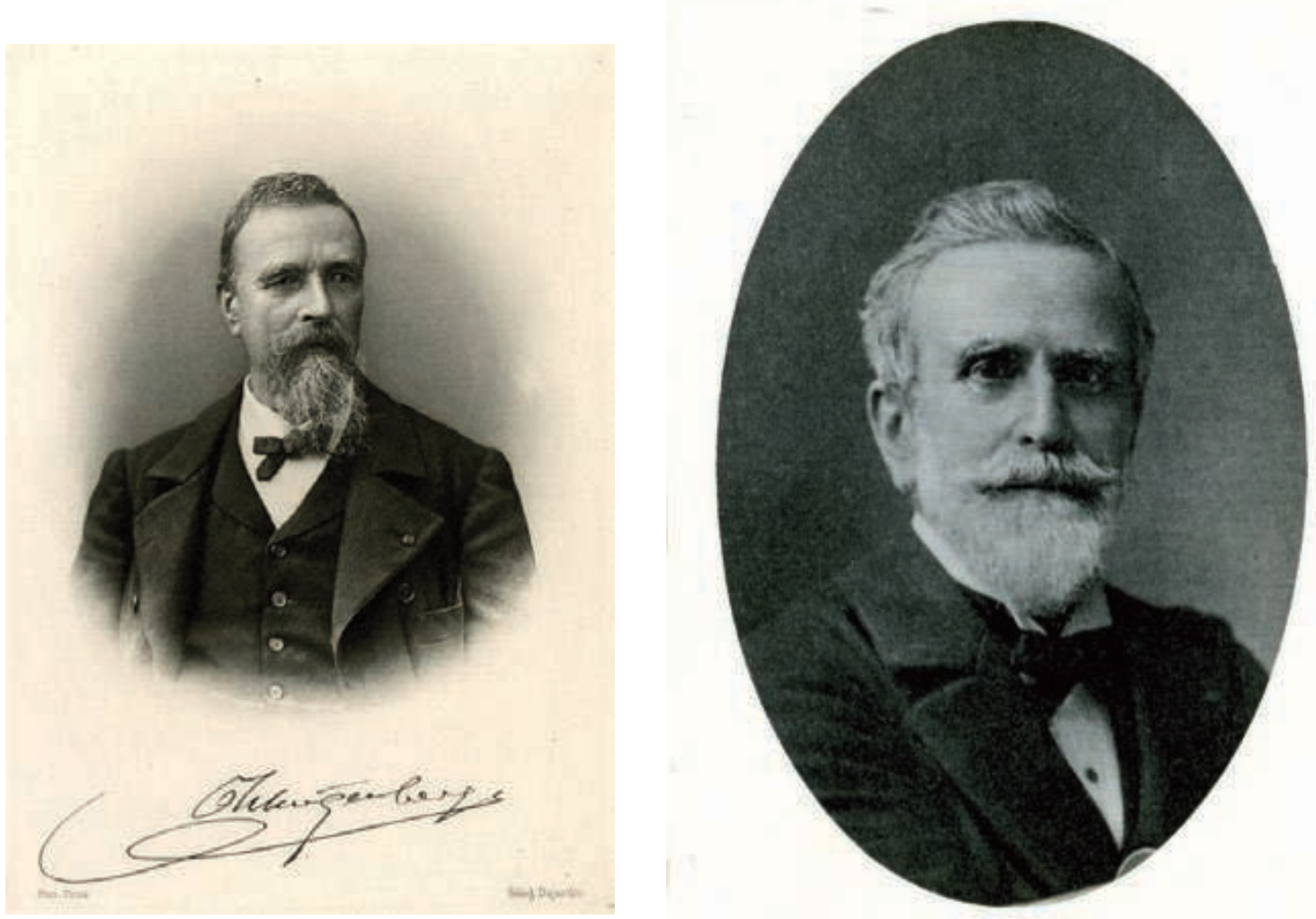

Paul Schützenberger (1829-1897) et Charles Lauth (1836-1913),
chimistes industriels, fondateurs de l'École municipale
de physique et de chimie industrielles (coll. ESPCI).

Est-ce que dans les bibliothèques des maîtres

fondateurs, il y avait beaucoup d'ouvrages ressortissant par exemple aux champs de la philosophie des sciences, de l'histoire et des sciences humaines et sociales, voire de la littérature ?

Avant la construction de cette bibliothèque, qui date des années 1930, l'École était installée dans les vieux bâtiments d'un ancien collège municipal. Il y avait une bibliothèque de prêt qu'on appelait une "bibliothèque roulante ". Est-ce que quelqu'un comme Bachelard y aurait eu sa place ? Nous n'avons pas les fiches " auteurs " et " matières " de cette époque, mais je ne le pense pas. La bibliothèque de l'ESPCI a un fonds spécialisé en physique, en chimie et en biologie. Dans ces disciplines, toutes les sous-disciplines sont représentées : de la physique du solide à l'optique, de la chimie analytique aux nano-sciences, des mathématiques pour ingénieur à la physique quantique, de la biochimie aux statistiques. Nos élèves ont un programme très intense à suivre et un grand nombre de livres de base à assimiler, ils ont déjà un solide programme de lectures. Quant aux chercheurs, ils doivent lire des quantités d'articles très techniques, le nombre d'articles scientifiques publiés par an augmentant de manière exponentielle, et il devient très difficile de suivre toute la bibliographie. Elèves et chercheurs trouvent cette documentation spécialisée le plus souvent assez facilement dans notre bibliothèque ou par l'intermédiaire des ressources du CNRS auxquelles ils ont accès également. Nous avons la chance de nous trouver dans un périmètre géographique où se trouvent plusieurs bibliothèques qui ont des fonds riches en philosophie, en histoire, en littérature. Si un étudiant cherche un roman, il peut se rendre facilement dans l'une d'entre elles. Nous avons cependant un rayon consacré aux livres de sciences et société et d'histoire des sciences, qui est un complément nécessaire à notre fonds spécialisé.

Ce que je viens de dire à propos de la spécialisation disciplinaire de notre fonds documentaire ne vaut cependant pas pour les bibliothèques personnelles des savants. Je prends pour exemple celle de Langevin, dont les descendants nous ont fait don de ses ouvrages. Il y a des livres scientifiques, mais pas seulement. La 
richesse de la bibliothèque de Langevin est à l'image de son œuvre et de son engagement. On y trouve, parmi les livres non scientifiques, des documents sur la Chine, sur l'éducation populaire, sur l'école laïque, des traités de psychologie, des livres d'économie, d'histoire et de philosophie, comme L'Intuition de l'instant de Gaston Bachelard, La Morale laïque et ses adversaires d'Albert Bayet, etc. Ces livres portent souvent une dédicace manuscrite des auteurs, comme Notre guerre, souvenir de résistance avec une dédicace d'Agnès Humbert : " $\mathrm{Au}$ professeur Paul Langevin, à Madame Paul Langevin, auxquels j'ai si souvent pensé pendant les années de notre guerre. Souvenir de ma respectueuse affection. 25/5/46".

Ce fonds a fait l'objet en 2002 d'un mémoire de maîtrise par un étudiant qui en a aussi réalisé le catalogage et l'indexation informatique. Nous avons un tirage papier du catalogue avec les textes des dédicaces.

\section{Dans quelle mesure cette bibliothèque pourrait-elle être un instrument de travail pour les historiens de la chimie et de la physique?}

Ceux qui travaillent sur le $20^{\mathrm{e}}$ siècle ou sur la fin du $19^{\mathrm{e}}$ peuvent trouver ici des livres, français ou anglais, aujourd'hui épuisés. Nous avons près de 200 thèses scientifiques d'avant 1900, la plus ancienne remontant à 1815. Notre bibliothèque est ouverte aux chercheurs extérieurs, et facilement accessible au cœur du $5^{\mathrm{e}}$ arrondissement de Paris. Quant aux archives, elles sont déjà connues et citées par les historiens, plusieurs livres et de nombreuses publications ayant vu le jour à partir de nos documents. Mais on peut certainement améliorer leur visibilité, par la numérisation d'une partie des collections notamment.

On peut trouver dans les archives des sujets de thèse ou de mémoire pour les étudiants en histoire des sciences, sur des sujets variés de la physique et de la chimie du $20^{\mathrm{e}}$ siècle. L'an dernier par exemple, une étudiante en master a fait son mémoire sur " la photographie industrielle " à partir des travaux de Charles Féry. Qui aujourd'hui se souvient que les travaux de la Commission LangevinWallon ont eu lieu dans cette bibliothèque? En 2009, nous avons organisé un séminaire sur le rôle de Langevin dans la réforme de l'enseignement, qui a permis de rappeler ces aspects. Plus récemment, un colloque sur les congrès de chimie Solvay a mis en lumière le rôle très important d'Albin Haller, chimiste alsacien, directeur de l'EMPCI, dans la chimie industrielle au niveau international. C'est ainsi, en organisant des journées thématiques, qu'on peut attirer des étudiants et chercheurs en histoire des sciences et donner vie et parole aux archives.

\section{C'est dans ce contexte qu'a été créé le Centre de ressources historiques de l'École?}

Le Centre de ressources historiques a été créé en 1984, sous l'impulsion des manifestations du centenaire de l'École. Avec l'appui du directeur, Pierre-Gilles de Gennes, grâce à la mobilisation des anciens et à la ténacité de l'ancienne conservatrice de la bibliothèque, Monique Monnerie, l'École s'est lancée dans une vaste entreprise de collecte d'archives.

Depuis sa naissance, ce centre accueille de nombreux chercheurs, essentiellement des historiens des sciences, des historiens de l'enseignement ou des sociologues qui travaillent sur des sujets très variés. Nous sommes aussi souvent sollicités par des éditeurs ou des particuliers à la recherche d'iconographie ou de renseignements à caractère historique. Le conservateur devient ainsi un historien des sciences " généraliste ", si l'on peut faire le parallèle avec le médecin généraliste. C'est ainsi que j'arrive à les orienter dans le fonds, sur des sujets aussi variés que la découverte de la radioactivité, le sonar, la physique du $19^{\mathrm{e}}$ siècle, la diffusion de la théorie de la relativité, la découverte des tubes à néon, les travaux de la commission Langevin-Wallon, l'engagement des scientifiques dans les mouvements pacifistes, l'histoire des polymères et de la chimie analytique, les Instituts Solvay, l'évolution de l'enseignement à l'ESPCI, les premiers professeurs et chefs de travaux, l'École pendant les deux guerres, ou encore sur les prototypes d'instruments conçus et réalisés ici et les familles de constructeurs, pour ne prendre que quelques exemples.

Avec ma spécialité d'historienne des sciences, je tente de montrer cependant, bien au-delà des grandes découvertes et des célébrités dont à juste titre cette " petite école municipale " peut s'enorgueillir, que la force de l'ESPCI se trouve aussi dans les synergies qui ont pu être développées entre des dizaines d'acteurs, célèbres ou anonymes, tous gens de grande inventivité dans leur science et de dévouement dans leur enseignement. C'est "l'influence du milieu ", dont Pierre Curie ${ }^{4}$ soulignait l'importance dans toute production scientifique. 
Est-ce que le Centre a reçu des fonds ayant appartenu à des professeurs ou anciens étudiants de l'École?

Oui, bien sûr. Grâce à la mobilisation de nombreux acteurs $^{5}$, les archives de Paul Langevin conservées par sa famille ont été transférées à l'ESPCI. Le premier versement a eu lieu en 1983. Le fonds est composé de 200 boîtes. Nous avons ensuite reçu les versements d'autres fonds : Georges Champetier, Albin Haller, Georges Claude, René Lucas, Pierre Biquard et bien d'autres, au total plusieurs dizaines de personnalités ayant un lien avec l'ESPCI. Ces fonds ont des volumétries variables, de quelques documents à des séries de boîtes, au total 200 boîtes supplémentaires. Je poursuis cette politique de collecte et de sauvegarde auprès des anciens élèves notamment, qui viennent régulièrement nous apporter des documents qu'ils ont en leur possession : photographies, notices, cahiers de cours, etc.

\section{Vous avez des documents tout à fait exceptionnels, des carnets de laboratoires et des cahiers d'expériences...}

Parmi ces documents, je voudrais m'arrêter sur un cahier de laboratoire qui me tient à cœur : celui de Charles Féry. Elève de la première promotion, chef de travaux puis professeur à l'EMPCI, Charles Féry fut un esprit très inventif. Il a conçu et fabriqué les prototypes de nombreux instruments de mesure et d'analyse chimiques par la voie optique (pyromètre optique, réfractomètre, spectromètre...), ainsi qu'une horloge électrique et une pile à dépolarisation par l'air qui a été utilisée pendant la Grande Guerre. Le cahier d'expériences consigne les résultats des travaux pratiques des élèves. Ces derniers, par groupes de deux ou trois, devaient réaliser une expérience sur un sujet donné par le professeur. Chaque expérience est décrite sur deux ou trois pages du cahier et signée par les élèves qui l'ont réalisée. Les sujets sont inspirés des travaux de recherche du professeur. De tels cahiers se prêteraient à des recherches historiques passionnantes dans leurs rapports avec les programmes d'enseignement, l'organisation des travaux pratiques et plus généralement, sur les liens entre enseignement et recherche.
Les cahiers d'expériences étaient donc copiés par les étudiants après le cours. Pouvez-vous nous parler plus précisément de ce fonds?

On a commencé très tôt à utiliser ce type de cahiers. Ils étaient fournis par l'École, ont le même format (un peu plus large qu'un in- $8^{\circ}$ ), et portent une étiquette : "École municipale de physique et de chimie industrielles ". Chaque élève inscrivait son nom sur la couverture. Cela paraît un peu scolaire, mais c'était ainsi à l'époque. Prenons par exemple le cahier de Paul Langevin quand il était élève : " $2^{\mathrm{e}}$ année, Manipulations de Physique. Étude d'un Moteur à Pression d'Eau, Système Schmid ". Il est parfaitement calligraphié à la plume à encre : " Ce moteur se compose d'un cyclindre dans lequel se meut un piston dont la tige actionne au moyen d'une bielle un axe horizontal muni d'un volant... ". Ce sont des notes sur les manipulations pour une séance de travaux pratiques, mises au propre par l'élève. Il y a aussi des schémas d'une grande précision, tracés à la plume, qui devaient demander beaucoup d'attention. Langevin est entré à 16 ans à l'École, il devait donc avoir 17 ou 18 ans quand il a écrit ce cahier. Il y a beaucoup de pages blanches entre les chapitres et très peu de ratures. Parfois, économies obligent, lorsqu'il reste beaucoup de pages blanches, Langevin utilise le même cahier pour une autre matière, en commençant l'écriture sur la dernière page après avoir retourné le cahier.

\section{Un tel manuscrit poserait presque les mêmes problèmes de déchiffrement, d'établissement du texte et d'interprétation que les manuscrits grecs?}

Le symbolisme a peu évolué et l'écriture reste parfaitement lisible par un lecteur d'aujourd'hui. La température est toujours représentée par $\mathrm{T}$, la pression par $\mathrm{P}$, le temps par $\mathrm{t}$, la vitesse par $\mathrm{v}$ ou $\mathrm{u}$, la force par F, etc. Ces notes servent à comprendre le cours. S'il s'agit d'un cahier d'expériences, ce sont alors les étapes de l'expérience et les résultats de mesure qui importent.

Il serait intéressant de mettre ces procédures en regard des connaissances scientifiques de l'époque. Est-ce que leur enseignement est précoce ou déjà largement partagé ? Les cahiers de notes de cours permettent de repérer le passage des innovations de la recherche à l'enseignement. Dans le domaine de la chimie organique, nous savons que les chimistes en France étaient partagés, jusqu'à la fin du $19^{\mathrm{e}}$ siècle, entre les atomistes (l'école de 
Wurtz) qui écrivaient les formules chimiques avec $C=12$ et $\mathrm{O}=16$, et les équivalentistes, comme Marcelin Berthelot qui écrivait $\mathrm{C}=6$ et $\mathrm{O}=8$ et qui considérait le benzène comme un triacétylène. Dans les cahiers de Langevin, on voit bien que les réactions chimiques sont écrites dans la notation atomique. On voit clairement les formules hexagonales des composés organiques, et les poids atomiques sont les mêmes qu'aujourd'hui. Rien d'étonnant, car les chimistes alsaciens que Langevin a eus comme professeurs avaient adopté très tôt la théorie et la notation atomiques.

Nous avons une autre série de cahiers surprenants de la main de Langevin. Il s'agit d'articles scientifiques de physique traduits de l'anglais ou de l'allemand - Langevin maîtrisait les deux langues. Ainsi par exemple le début d'une traduction de la Theory of sound : " La théorie du son par John William Strutt, baron Rayleigh ". Dans un autre cahier, nous avons un article fondamental d'Hermann Minkowski (ill. p. 54), dans lequel son auteur introduit la notion d'espace-temps à quatre dimensions : "Les équations fondamentales des phénomènes électromagnétiques dans les corps en mouvement, traduit de l'allemand $"^{6}$. Pourquoi Langevin faisait-il ces traductions ? Pour ses élèves ? En vue d'une publication? Dans les deux cas, on peut y voir un désir de diffusion des connaissances, car nous savons que Langevin aimait partager la science avec les autres, qu'ils soient étudiants, scientifiques ou simples curieux.

Dans le même temps, ces traductions et autres notes bibliographiques témoignent de la connaissance que Langevin avait de la bibliographie internationale sur les sujets à la pointe de la recherche. L'examen des cahiers de cours, des notes et des traductions peut aider à suivre l'évolution de sa pensée scientifique et à comprendre le cheminement qui l'a conduit à adopter, avant beaucoup d'autres, la relation d'Einstein entre masse et énergie, par exemple. De tels documents mériteraient d'être étudiés dans une perspective interdisciplinaire, prenant en compte aussi bien la bibliographie matérielle que les contenus scientifiques...

\section{Ces archives sortent donc du cadre spécialisé de la physique et de la chimie...}

On trouve dans ces archives Langevin des documents relatifs à ses voyages, comme ceux qu'il a faits lorsqu'il était à l'École Lavoisier, école primaire supérieure où il a préparé le concours d'entrée à l'EMPCI. La correspondance scientifique est elle aussi d'un grand intérêt : elle relie Langevin aux plus grands savants de l'époque. On a aussi beaucoup de ses brevets...

\section{La bibliothèque conserve-t-elle ces brevets scientifiques?}

Le dépôt de brevets est une tradition à l'ESPCI depuis ses origines. Ceux de Langevin sur le sonar sont les plus connus. Georges Claude, fondateur d'Air Liquide avec son camarade de promotion Paul Delorme, avait déposé des centaines de brevets. Certains exemples sont moins connus, comme celui du bec Méker, un petit instrument ordinaire qui se trouve en nombre dans tous les laboratoires de chimie, et qui a été breveté par Georges Méker, élève de la $12^{\mathrm{e}}$ promotion. On pourrait en citer des dizaines d'autres. Les chercheurs de l'ESPCI ont toujours été des inventeurs d'instruments, des artisans de la science, pour reprendre l'expression utilisée dans l'ouvrage de Denis Baudouin ${ }^{7}$, car il fallait jadis inventer l'instrument adapté pour faire de la recherche, alors qu'aujourd'hui il est le plus souvent fabriqué en série et acheté sur catalogue. La culture et le savoir-faire en matière de dépôt des brevets se poursuivent aujourd'hui à l'ESPCI plus que jamais, et de nombreuses start-up sont créées, fruits de la recherche et de l'innovation conduites ici.

\section{Propos recueillis par Christian Jacob}

\section{Notes}

1 - Ils ont donné lieu à une première publication dans un numéro spécial du Bulletin de la Société industrielle de Mulhouse : La chimie et l'Alsace $\left(\mathrm{n}^{\circ} 2,1994\right)$.

2 - On appelle ainsi des revues électroniques en libre accès, avec comités de lecture, alimentées par les articles déposés dans les archives ouvertes telles que HAL ou ArXiv, et non publiés par ailleurs.

3 - Information scientifique et technique

4 - « Dans toute production scientifique, l'influence du milieu dans lequel on travaille a une importance très grande et une partie des résultats est due à cette influence... Les professeurs de l'École de physique et de chimie, les élèves qui en sortent, constituent un milieu bienfaisant et productif qui m’a été très utile ". (Pierre Curie, conférence à la Sorbonne, 1904)

5 - Voir à ce sujet Bernadette Bensaude-Vincent et Christine Blondel, Les archives de Paul Langevin à l'École de physique et de chimie industrielles, in La Gazette des archives, $\mathrm{n}^{\circ} 145,1989$, p. 150-153

6 - On peut consulter ce document en ligne sur l'archive ouverte HAL.

7 - Denis Beaudouin, Charles Beaudouin, une histoire d'instruments scientifiques, Paris, EDP Sciences, 2005 